\title{
Educational software evaluation research: Balancing internal, external, and ecological validity
}

\author{
SARAH RANSDELL \\ New College of the University of South Florida, Sarasota, Florida
}

\begin{abstract}
The difficulties inherent in the evaluation of educational software are described in terms of the tradeoffs between internal, external, and ecological validity. Larger issues in evaluation research design and computer-based instruction are highlighted by primary and metaanalytic studies designed to reveal the effects of computer simulations in psychology classrooms and laboratories. The effectiveness of classroom and laboratory computer activities depends on how the inclusion of software, as well as the evaluation process itself, changes the entire instructional process.
\end{abstract}

Educational software is proliferating at a tremendous rate. Unfortunately, the systematic evaluation of software's effects on learning is not growing nearly as rapidly. Evaluation design difficulties can contribute to the disparity between growth in the development and evaluation of educational software. Many design obstacles are shared by all evaluation research, such as questions of validity and the nature and scope of outcome measures. Others are unique to the demands of media research within the college classroom, including instructor-as-observer and student-as-respondent biases. In this paper, I will describe factors that one must consider when weighing the requirements of internal, external, and ecological validity in evaluation research.

\section{Evaluation Research and Validity}

Internal validity refers to the degree to which a design allows for unconfounded results. The control afforded by random assignment to groups, as well as the tight definition of independent and dependent variables, greatly improves internal validity, but it often limits generalizability to natural contexts. External validity is the degree to which results can be generalized to apply to other populations, settings, or levels of variables. The use of large, heterogeneous samples improves external validity, but it is difficult to implement in educational situations. Both types contribute to the knowledge that software demonstrably improves actual learning.

Problems of design such as unpredictable student attrition within and between groups may jeopardize internal validity; yet such attrition is often representative of real classroom conditions. Changes other than the simple addition of software-changes, for example, in the contents of the course syllabus-are common in the classroom, but

Send correspondence and requests for reprints to the author at the Division of Social Sciences, New College, Sarasota, FL 34243-2197 (e-mail: dsdaiam@cfrvm.bitnet). they can make interpretation difficult. Nowhere does this problem of control versus generalizability occur more seriously than in naturalistic experimentation (see Banaji \& Crowder, 1989).

Many researchers claim that experimental research requires conditions simply not attainable in evaluation contexts (Borich \& Jemelka, 1981). One difficulty lies in the disentanglement of interactions inherently present in the classroom. Learning is necessarily a function of many factors, some of which are under the instructor's control, and others of which are under the student's. Experimental designs, on the other hand, afford great control over most stimulus parameters. A second concern is the problem of withholding potentially beneficial programs from some students, a particular dilemma in between-group comparisons. The following metaanalyses of computerbased instruction should serve to amplify and summarize the difficulties found in individual evaluation projects.

\section{Metaanalytic Evaluation Research}

Metaanalysis of the effects of educational software is well suited to reveal potential confounds caused by limitations in design. This technique combines effect sizes of individual studies (expressed as the average distance between experimental and control groups) into one common effect size (see Glass, McGaw, \& Smith, 1981). It has proven invaluable to evaluation research because it can partition the effect sizes of multiple factors on learning.

For example, C. L. Kulik, J. A. Kulik, and Cohen (1980) conducted a metaanalysis to compare 59 collegelevel computerized and conventional courses. The criteria for inclusion in the metaanalysis were that the study had to be conducted in an actual college classroom, to include quantitative outcome measures, and to have fair controls between treatment groups. These studies contained both computer programs replacing lectures and those merely supplementing them. An effect size of .51 was found in favor of computerized courses, but only .13 when teacher was not crossed with medium of instruction. 
Across all studies, a quarter standard deviation improvement (.25 effect size) was found for the computerized courses.

In an update, J. Kulik and C. Kulik (1987) again found generally positive effects of the use of educational software in the classroom. Final exam scores showed an overall effect size of .31. The results, however, varied as a function of the evaluation design. The effects were significantly stronger in published studies, when instructor was not crossed with medium, in more recent studies, in studies of shorter duration, and for college-level students. In sum, editorial gatekeeping and experimental design choices may account for many of the "advantages" of computer-based instruction.

In their most recent work, C. Kulik and J. Kulik (1991) conducted a metaanalysis of 254 controlled evaluation studies and found that computer-based instruction had positive effects. These studies were free from substantial differences in the aptitudes of the treatment and control groups, from unfair teaching of the criterion test to one group, and from different rates of attrition between groups. Consistent with earlier metaanalyses, the effects were larger for published studies, in which the teachers were different in each medium, and for short-duration studies.

Metaanalyses have also shown that the evaluation of software "out of context" is problematic. Jolicoeur and Berger (1986) did a metaanalysis of studies which measured the effects of software programs administered to individuals outside of college courses. They focused on commercial products available for evaluation and concluded that these had poor reliability and validity mainly because the programs were not used to evaluate actual courses. Jolicoeur and Berger therefore stress the need for teachers to conduct naturalistic studies of software as they are used in genuine classroom contexts.

\section{Evaluation Research Between Media}

Evaluation studies generally compare computer-based instruction with traditional forms of teaching, such as lectures. However, some researchers have claimed that there is an indeterminacy to between-medium comparisons because "when only the least significant aspects of instruction are allowed to vary, nothing of interest could, and did, result" (Saloman \& Clark, 1977). After reviewing current research, Hagler and Knowlton (1987) concluded that comparing media, rather than messages (the symbols which carry meaning) poses a serious threat to internal validity. Studies documenting superior learning with computer software often contain confounds. For example, extra study time, rather than unique characteristics of the software, may improve learning in computer media conditions (Jamison, Suppes, and Welles, 1974).

Clark (1983) suggests that media comparison problems are driven by the fact that media are merely delivery devices and that significant advantages result from uncontrolled factors besides the medium. He reports metaanalyses that reveal correlations between medium and achievement, but they appear to be confounded by differences in instructional method between computer and noncomputer-based instruction. A novelty (Hawthorne) effect for newer media was noted (see also Niemiec \& Walberg, 1987, for a narrative review).

Two between-medium examples further illustrate this problem. In a well-controlled comparison of a computer activity and a traditional lab exercise on the same topic, Belmore (1983) found no significant differences between groups on any qualitative measure. Three dependent variables were included-mean recall performance, knowledge questions, and student evaluation ratings. The only reliable effect was that traditional labs took longer to complete than the computerized labs. Welsh and Null (1991) also used a between-medium comparison but with different instructors across media. Even then, no significant differences in mean knowledge scores were found between groups. If given an assignment afterwards, both groups did better. Welsh and Null concluded that computer instruction should be considered in combination with instructor factors. Educational software may produce beneficial effects, but it is unlikely to do so without changes in the teacher's instructional methods as well.

\section{An Evaluation Project}

Clearly, we need to know more about the potential threats to validity inherent in the evaluation of educational software. The following section describes an evaluation project that highlights some of the tradeoffs between internal, external, and ecological validity factors. A set of 13 topics in three sections of an introductory psychology course was either presented in traditional lecture format alone or supplemented by computer activities shown via a projection system during class. Ninety students in 8-week summer terms at a community college evaluated the software on self-report questionnaires. The majority worked full time while taking this course and had experience of computers mainly from their jobs. Most were 1 st or 2nd-year students who were taking the class primarily because it was a college requirement. The students were not aware that their professor was the author of the software or that such evaluation measures were not regularly a part of the class.

The regular syllabus covering 12 chapters was used, and the students responded to both a midterm and an endof-term questionnaire. A 1st-day questionnaire asked general demographic questions and requested the level of previous experience in using computers. The midterm questionnaire included anonymous, open-ended questions about what the students liked and disliked about the computer activities (see Appendix A).

Appendix A provides a summary of modal responses to questions asked at midterm about the computer activities and the course in general. The responses from the community college students are compared with those of 35 students at an honors college who participated in the same computer activities as part of a weekly lab rather than as a supplement to class lectures. Both the positive 
and the negative comments reflect how the entire instructional method and not simply unique characteristics of the computer medium affected the course. Several examples drawn from the written comments illustrate this point. The first set comes from classroom use by the community college students. "I like the use of real-world examples. It might be easier to do some of the activities as an individual than in a group. The computer activities give a new perspective to text and lecture. I like being tested and comparing my results with the 'norm.' "Some comments clearly indicate the interaction of instructor guidance and computer activity. "Your explanation of what's happening makes it easier. The main objective is clear as long as you restate it." The honors college students also commented on this with respect to their labs. "If something was unclear, you could ask the TA." The community college students felt that the lectures were often too fast paced. "Computer activities give you time to relax and understand the material." And honors students felt that "computer coverage is more in depth than time allows in lecture." Some honors students felt that the computer activities were too simple: "Very basic, and often repetitive treatment of content." The community college students rarely commented on the simplicity of the simulations but tended to focus on how their structure improved comprehension: "Objectives were always found in the introduction and repeated in the conclusions."

The end-of-term questionnaire asked for anonymous ratings of how the computer activities compared with the lecture coverage in terms of involvement, understanding, and interest (see Appendix B). Overall, the ratings were positive for both media. Some exceptions were that students felt rushed (Question 7) and that too much material was covered (Question 8), which is not surprising for an 8-week summer term and corroborates the midterm data. Relative ratings were actually of more interest than $a b-$ solute, so paired comparison $t$ tests were conducted between instructional methods. An alpha of .01 divided by 12 comparisons, or .001 , was used to determine significance. Question 8 showed that computer activities were significantly "less dense"' than lecture coverage $[t(94)=$ 3.59], which was probably a result of less time spent on the computer activities overall. Responses for two of the questions, about in-class participation [Question 5, $t(93)=6.24]$ and class activity level (Question 10, $t(98)=7.80]$, were significantly more positive for computer presentation than for lecture alone. Computer activity also received significantly higher ratings for "fun" [Question 6, $t(93)=3.17$ ] and "interest level" [Question $11, t(95)=4.08$ ]. It is likely that novelty and relative time spent with each method contributed to these differences in enjoyment and interest.

\section{Threats to Validity: Some Conclusions}

Questionnaire data from the present project suggest that an effect of instructional method may contribute to classroom learning at least as much as any specific effects of the software. Computer simulations increase classroom activity and are interesting in part because unlike classroom lectures they offer a welcome novelty. Although the present project was not designed as a direct comparison of lectures and software, it did suggest many difficulties inherent in such comparisons. Table 1 summarizes some factors that threaten internal, external, and ecological validity. Factors mainly under student control tend to limit internal validity; they include attrition, improvements or declines in performance due to students' attendance or study habits, and variable interest in, and difficulty of, individual topics. Between-medium comparisons are likely to include order effects such as novelty and recency effects, especially over the length of an entire course. Furthermore, descriptions taken from questionnaires must rely on verbal reports as data; even anonymous comments are subject to social desirability among other response biases. The needs of a particular class are likely to require changes not anticipated when one is designing an evaluation study, such as extra-credit work, reviews of studentgenerated questions, and pacing the material along with students' progress. Perhaps one of the greatest threats to internal validity is the problem of operationally defining the independent variable of the medium. A multitude of factors affect classroom learning; computer activities can supplement learning, but they do not do so in isolation.

Threats to external and ecological validity include the problem of isolating how an instructor implements a computer activity during class and his/her general teaching style. Recall that metaanalytic studies revealed problems with designs in which instructor was crossed with a medium. Instructors have at least two objectives when they evaluate instructional methods. First, they intend that students learn as much as possible in their courses. Second, they want to know what impact new methods, such as computer simulations, might have on this learning. Evalu-

Table 1

A Summary of Factors That Threaten Validity Internal Validity Factors

uneven rates of student attrition over time improvement on exams with practice alone student-selected class attendance, meeting times unavoidable interactions (i.e., attendance $x$ performance) variable interest in topics

variable difficulty of topics novelty, order, recency effects

verbal reports as data (e.g., use of questionnaires) necessary adaptations over time (i.e., extra credit, reviews) definitions of independent variable (i.e., "'media")

External and Ecological Validity Factors

isolating method from general teaching style evaluation while evaluating knowledge studies of short duration studies conducted outside the classroom homogeneous samples of college student ability limited computer resources bias of publishing "positive" results general instructor as observer biases general student as respondent biases 
ation of methods made while one is evaluating knowledge may be difficult, since research designs must remain fixed over time and students' needs may necessitate changes in design. The need for some degree of flexibility in evaluation design limits internal validity and yet improves the degree to which such results can be generalized to actual classroom settings.

Studies of short duration, those which cover less than an entire course, are clearly less generalizable, as are those which are conducted outside the classroom setting. Differences between midterm questionnaire comments of community college and honors college students point to the problem of using homogeneous samples. Evaluators as instructors are limited by the sample of students whom they teach and to the computer resources that they have available. Classroom presentation equipment and laboratory microcomputers often dictate where software can be tested. Finally, a priori expectations on the part of both the instructor and the student can limit clear interpretations of evaluation data. Instructors are motivated to find positive results in order to publish them. Students are motivated to get positive results so that they will succeed in the course.

Some researchers have argued that the major contribution of evaluating computer-based instruction may be simply having the instructor assess and modify existing courses (Hagler \& Knowlton, 1987). Castellan (1986) provides a guide to instructors for evaluating and selecting courseware and especially for effectively introducing its use. In this guide, he supports the view that evaluation should include the use of the software in a classroom environment with a large-screen monitor or projection system. How the instructor uses educational software determines results as surely as the software itself does. Balancing the requirements of internal, external, and ecological validity, as well as viewing software as part of a larger effect of instructional method, is a difficult task. However, further progress will be required for us to successfully evaluate the effects of educational software on learning. And successful evaluation is tantamount to widespread acceptance and use among college faculty.

\section{REFERENCES}

Banaj, M., \& Crowder, R. (1989). The bankruptcy of everyday memory. American Psychologist, 44, 1185-1193.

BELMORE, S. M. (1983). RELEASE FROM PI: Comparison of traditional and computer modules in an experimental psychology laboratory. Behavior Research Methods \& Instrumentation, 15, 191-194.

BorICH, G., \& JemelKa, R. (1981). Evaluation. In Computer-based instruction: A state-of-the-art assessment (pp. 161-209). New York: Academic Press.

Castellan, N. J., JR. (1986). Issues in the effective use of computers in introductory and advanced courses in psychology. Behavior Research Methods, Instruments, \& Computers, 18, 251-256.

ClARK, R. E. (1983). Reconsidering research on learning from media. Review of Educational Research, 53, 445-459.

Glass, G., McGaw, B., \& SMITH, M. (1981). Meta-analysis in social research. Beverly Hills: Sage.

HAGLER, P., \& KNOWLTON, J. (1987). Invalid implicit assumption in CBI comparison research. Journal of Computer-Based Instruction, 14, 84-88.
Jamison, D., SupPes, P., \& WELles, S. (1974). The effectiveness of alternative instructional media: A survey. Review of Educational Research, 44, 1-68.

Joucoeur, K. \& BERGER, D. E. (1986). Do we really know what makes educational software effective? A call for empirical research on effectiveness. Educational Technology, 26, 7-11.

KulıK, C. L., Kulik, J. A. (1991). Effectiveness of computer based instruction: An updated analysis. Computers in Human Behavior, 7, $75-94$.

Kulik, C. L., Kulik, J. A., \& CoHEN, P. (1980). Effectiveness of computer based college teaching: A meta-analysis of findings. Review of Educational Research, 50, 525-544.

Kulik, J. A., \& KuLIK, C. L. (1987). Review of recent research literature on computer based instruction. Contemporary Educational Psychology, 12, 222-230.

Niemiec, R., WALBERG, H. (1987). Comparative effects of computerbased instruction: A synthesis of reviews. Journal of Educational Computing Research, 3, 19-37.

SALOMAN, G. CLARK, R. (1977). Reexamining the methodology of research on media and technology in education. Review of Educational Research, 47, 99-120.

WELSH, J. A., NuLl, C. H. (1991). The effects of computer-based instruction on college students' comprehension of classic research. Behavior Research Methods, Instruments, \& Computers, 23, 301-305.

\section{APPENDIX A \\ Summary of Negative $(-)$ and Positive $(+)$ Comments on the Midterm Questionnaire by Community College (CC) and Honors College Students (HC)}

1. When watching the computer activities, were you ever frustrated by them? If so, describe briefly the reason for your frustration.

*CC(-)-went too fast, too slow, difficult to see the screen if in back, book definitions sometimes different, might be easier as an individual than as a group to do some of the activities.

${ }^{*} \mathrm{CC}(+)-c o m p u t e r$ shows what's happening, broader and different view of material, like the use of real-world examples, interesting, made text come to life, helpful to understanding the text.

${ }^{*} \mathrm{HC}(-)$-very basic treatment, more work than I thought at first, sometimes repetitive, not always obvious what I was supposed to be learning, counterintuitive commands, navigation problems, sometimes directions not explicit, frustrating analyzing own results.

*HC(+)-reinforces simple topics, helps retain information, need to view examples first.

2. Was the computer display on the screen always easy to see? If not, which displays were problematic and how often?

${ }^{*} \mathrm{CC}(-)$-your explaining of what's occurring makes it easier, went too fast, need to be front and center, lights should be dimmed, too dark to take notes, screen too small.

${ }^{*} \mathrm{CC}(+)$ - easy to see, especially with lights out, need to have a good seat and you're ok.

${ }^{*} \mathrm{HC}(-)$-some color combinations were harder to see, letters sometimes too small.

*HC(+)-generally user-friendly.

3. Did you usually understand the computer program's main objective? That is, what it was all about? If not, which activity was difficult to see what it was for?

$* \mathrm{CC}(-)$-schedules of reinforcement one was confusing, ok as long as you restate it, made more sense with practice, went too quickly sometimes. 
${ }^{*} \mathrm{CC}(+)$-professor restated objectives, very straightforward, helped tie discussion to lecture notes.

${ }^{*} \mathrm{HC}(-)$-very simple level, reinforcement one was difficult, sometimes it didn't explain why answers were wrong.

${ }^{*} \mathrm{HC}(+)$-always outlined in intro and in conclusions, if not clear could ask the TA, usually easy and right away.

4. Are the concepts and terminology in the computer activities related to the material covered in the class lectures? If not, how often is this a problem?

* $\mathrm{CC}(-)$-usually similar if not the same, lecture definitions go more quickly.

${ }^{*} \mathrm{CC}(+)$-they reinforce one another, differences make class more interesting, make more memorable because repeated.

${ }^{*} \mathrm{HC}(-)$-often seemed too basic, sometimes not but were all in computer glossary, should cover more than 1 topic, should be discussed before lab in lecture.

${ }^{*} \mathrm{HC}(+)$-complementary, computer coverage more in depth than time allows in lecture, key terms helpful.

5. Were any of the activities tedious or boring? Which ones?

* $\mathrm{CC}(-)$-weren't enough of them to be boring, better if in color, some very boring, some too long, more than $10 \mathrm{~min}$ is tedious.

${ }^{*} \mathrm{CC}(+)$-helped explain subjects, different from other classes, gave a new perspective, conclusions were the most helpful part.

${ }^{*} \mathrm{HC}(-)$-questions were repetitive, specific content sometimes boring, boring if just have to hit the space bar, boring if confusing, superficial coverage is more boring, all of them but that's the way to learn.

${ }^{*} \mathrm{HC}(+)$--they go pretty quickly, those with more involvement are more fun, reading from screen is not enough, good learning tools.

6. Were any of the activities particularly interesting? Which ones?

*CC-most comments were content-specific, statements about liking activity during class, participation, exercise and other action words appeared.

*HC-simplified are less interesting, less activity is less interesting, liked being "tested," personal significance is more interesting.

7. Please add any other comments you'd like to make about the computer activities or the course in general.

*CC-gave you time to relax and understand material relative to when lecturing, course was too fast-paced, easier to remember the activity along with notes.
*HC-need more discussion during class, enjoy activity, add more graphics, add more questions, good overview and pretest, nice to take a break from class learning, activities coordinated with class.

\section{APPENDIX B \\ Mean Ratings Across All Three Sections on the End of Term Questionnaire $(N=56)$}

Rating Scale

$1=$ strongly agree

2 = somewhat agree

3 = neither agree nor disagree

4 = somewhat disagree

5 = strongly disagree

Lecture Computer

1. This type of instruction improved my understanding of the material.

$1.50 \quad 1.68$

2. I learned what material was most important during this type of instruction.

3. This type of instruction made the material easier to understand.

$1.74 \quad 1.68$

4. I enjoyed the way topics were covered by this type of instruction.

5. This type of instruction helped class participation and involvement.

6. Subject matter was fun to learn about during this type of instruction.

7. I felt rushed during this method of instruction.

8. Too much material was covered during this method of instruction.

$2.73 \quad 3.41 *$

9. I remember information better when it's covered by this method of instruction.

$\begin{array}{ll}2.05 & 1.78\end{array}$

10. The level of class activity was higher during this method of instruction.

11. This type of instruction was usually interesting to me.

12. Overall, I think this method of instruction should be used with other students taking this course in the future.

$2.07 \quad 1.46^{*}$

${ }^{*} \alpha=.001$ 